RICYDE. Revista Internacional de Ciencias del Deporte doi: $10.5232 /$ ricyde

Rev. int. cienc. deporte

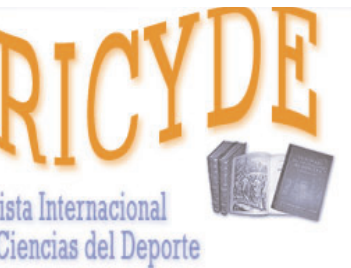

RICYDE. Revista Internacional de Ciencias del Deporte VOLUMEN XI - AÑO XI

Páginas:297-304 ISSN:1885-3137

Número 41 - Julio - 2015

\title{
Perfeccionismo, afectividad y satisfacción con la vida en educación física Perfectionism, affectivity and life satisfaction in physical education
}

\author{
Antonio Méndez-Giménez, José-Antonio Cecchini-Estrada, Javier Fernández-Río \\ Universidad de Oviedo. Asturias (España)
}

\begin{abstract}
Resumen
El perfeccionismo en la adolescencia ha sido asociado a altos niveles de desajuste y trastorno psicológico y bajos niveles de bienestar subjetivo y ajuste psicológico. Pero, no todas las dimensiones del perfeccionismo son insanas y desadaptativas. Mediante un análisis path se exploró un modelo para determinar cómo son las relaciones entre las dimensiones del perfeccionismo (autoexigencia y presión externa), la afectividad (positiva y negativa) y la satisfacción con la vida, en el contexto de la educación física. Participaron 388 estudiantes de $1^{\circ}$ a $4^{\circ}$ de Secundaria (222 varones y 166 mujeres; $M=14.24 \pm 1.45$ años). La autoexigencia predijo de manera positiva y directa la satisfacción con la vida; el afecto positivo también medió esta relación. La presión externa predijo directa y negativamente la satisfacción con la vida, cuya relación fue también mediada por el afecto negativo. Los resultados confirman una dimensión saludable de perfeccionismo (autoexigencia) y otra no saludable (presión externa) en la adolescencia.
\end{abstract}

Palabras clave: bienestar; calidad de vida; salud mental; afectividad

\begin{abstract}
Perfectionism during adolescence has been associated to high levels of psychological unbalance and distress, and low levels of subjective wellness and psychological adjustment. However, not all dimensions of perfectionism are negative and maladaptive. A path analysis was used to explore a model to determine the relations between two dimensions of perfectionism (Self-imposed and External pressures), affectivity (positive and negative) and life satisfaction in physical education. 388 1st to 4th secondary students (222 males and 166 females; $M=14.24 \pm 1.45$ years) agreed to participate. Self-imposed positively predicted life satisfaction, while positive affect mediated this effect. External pressures negatively predicted life satisfaction and negative affect mediated this effect. Results confirm a healthy dimension of perfectionism (self-imposed), and an unhealthy dimension (external pressures) in adolescence.
\end{abstract}

Key words: wellness; quality of life; mental health; affectivity. 
Méndez-Giménez, A.; Cecchini-Estrada, J.A.; Fernández-Rio, J. (2015). Perfeccionismo, afectividad y satisfacción con la vida en educación física. RICYDE. Revista internacional de ciencias del deporte, 41(11), 297304. http://dx.doi.org/10.5232/ricyde2015.04107

\section{Introducción}

$\mathrm{E}$ perfeccionismo es una disposición de la personalidad caracterizada por esfuerzos de impecabilidad, establecimiento de altos estándares de rendimiento y una tendencia a excesivas evaluaciones críticas (Frost, Marten, Lahart, y Rosemblate, 1990; Hewitt y Flett, 1991, 2002; Stoeber y Childs, 2011). Se trata de un rasgo que impregna todos los ámbitos de la vida (particular, laboral y escolar) y puede afectar a la propia apariencia personal y a las relaciones sociales (Stoeber y Stoeber, 2009).

Tradicionalmente, el perfeccionismo ha sido relacionado con diversas patologías: estrés (Hewitt y Flett, 1991), depresión y ansiedad (Kawamura, Hunt, Frost, y DiBartolo, 2001), trastornos de alimentación (Cockell y col., 2002), ideación suicida (Chang, 2002), fobia social (Arana, 2003), paranoia, psicoticismo, desórdenes psicosomáticos y trastorno obsesivo compulsivo (Frost, Heimberg, Holt, Mattia, y Neubauer, 1993). Sin embargo, en la actualidad la diferenciación de dos dimensiones principales de perfeccionismo ha permitido precisar estas relaciones (Stoeber y Otto, 2006). La dimensión esfuerzos perfeccionistas (estándares personales perfeccionistas o perfeccionismo auto-orientado) ha sido asociada con características, procesos y resultados positivos, como afrontamiento adaptativo, afecto positivo, bienestar subjetivo y ajuste psicológico (Einstein, Lovibond, y Gaston, 2000). En cambio, la dimensión preocupaciones perfeccionistas (preocupación por los errores, dudas acerca de las acciones, preocupación por la evaluación de los demás, y sentimientos de discrepancia entre las expectativas y el rendimiento), equiparada al perfeccionismo socialmente prescrito, ha sido asociada con características, procesos, y resultados negativos como el neuroticismo, el afrontamiento desadaptativo y el afecto negativo, así como a niveles altos de indicadores de desajuste y el trastorno psicológico (Einstein y col., 2000).

En la adolescencia, las preocupaciones perfeccionistas se asocian con mayores niveles de miedo al fracaso, estrés, depresión, ansiedad y enfermedades, y bajos niveles de confianza académica y satisfacción con la vida (Hewitt y col., 2002; Nounopoulos, Ashby, y Gilman, 2006; Stoeber, y Rambow, 2007). Por el contrario, la dimensión esfuerzos perfeccionistas ha sido asociada con indicadores de bienestar subjetivo y ajuste psicológico, por ejemplo, mayores niveles de esperanza de éxito, motivación para asistir a la escuela y preparar exámenes, dominio y orientación del trabajo (mostrando una preferencia por tareas difíciles), confianza académica, aceptación de los pares, número de horas dedicadas a estudiar a la semana, y logro académico (indicado por promedio más alto en las calificaciones), así como con una mayor autoestima y satisfacción con la vida (Hewitt y col., 2002; Nounopoulos y col., 2006; Stoeber y Rambow, 2007).

El estudio de Gaudreau y Thompson (2010) asoció de manera diferente las dimensiones del perfeccionismo con indicadores adaptativos y desadaptativos generales (afecto positivo y afecto negativo, respectivamente) e indicadores adaptativos del dominio general y específico (satisfacción con la vida y académica). El afecto positivo se caracteriza por alta energía, concentración completa y dedicación agradable, mientras que el afecto negativo refleja un estado emocional que incluye ira, culpabilidad, temor y nerviosismo (Sandin, 2003). La satisfacción con la vida se define como una valoración global que la persona hace sobre su vida, comparando lo que ha conseguido, sus logros, con lo que esperaba obtener, sus expectativas (Cabañero y col., 2004). 
Méndez-Giménez, A.; Cecchini-Estrada, J.A.; Fernández-Rio, J. (2015). Perfeccionismo, afectividad y satisfacción con la vida en educación física. RICYDE. Revista internacional de ciencias del deporte, 41(11), 297304. http://dx.doi.org/10.5232/ricyde2015.04107

Lozano, García, Martín, y Lozano (2012) han validado al castellano el Inventario de Perfeccionismo Infantil (IPI) a partir de las escalas Hewitt Multidimensional Perfectionism Scale (HMPS) (Hewitt y Flett, 1991) y Adaptative/Maladaptative Perfectionism Scale (AMPS) (Rice, y Preusser, 2002) y encontraron una estructura tridimensional del perfeccionismo. La dimensión Autoexigencia se refiere a la actitud perfeccionista con que el niño afronta sus tareas y, en opinión de los autores, se corresponde con el Perfeccionismo Auto-orientado. La Presión externa alude a la percepción de un entorno que demanda conductas perfeccionistas y se corresponde con el Perfeccionismo Prescrito Socialmente. La dimensión, Autovaloración, agrupa los ítems de tres dimensiones del AMPS: Sensibilidad a los errores, Necesidad de admiración y Autoestima contingente.

En el ámbito de la Educación Física (EF), Méndez-Giménez, Cecchini y Fernández-Río (2015) comprobaron un modelo en el que la autoexigencia predijo positivamente las cuatro metas de logro, mientras la presión externa predijo, negativamente, las metas de aproximación-maestría $\mathrm{y}$, de manera positiva y directa, la motivación controlada y la desmotivación. Esta última también fue mediada por las metas de aproximación-maestría. Asimismo, las metas de aproximación predijeron positivamente la motivación autónoma, y las de rendimiento, la motivación controlada.

Dado que la investigación sobre perfeccionismo adolescente ha sido prácticamente realizada con sujetos de otras culturas o nacionalidades (excepto el estudio de Méndez-Giménez, Cecchini-Estrada, y Fernandez-Río, 2015), el objetivo de este trabajo es explorar cómo es la relación entre las dos primeras dimensiones del perfeccionismo (autoexigencia y presión externa), el afecto (positivo y negativo) y la satisfacción con la vida en una muestra de estudiantes adolescentes en el contexto de la EF. El modelo representado en la Figura 1 se puso a prueba mediante un path análisis.

\section{Método}

\section{Participantes}

Participaron un total de 388 adolescentes de 6 institutos de 1 a $4^{\circ}$ de Educación Secundaria del Principado de Asturias; 222 varones (con un media de edad $=14.17 \pm 1.45$ años, rango $=$ $12-17$ ) y 166 mujeres (con un media de edad $=14.28 \pm 1.49$ años, rango $=12-17$ ).

\section{Instrumentos}

Perfeccionismo. Se emplearon dos subescalas del Cuestionario de Perfeccionismo Infantil (IPI; Lozano y col., 2012) adaptadas a la EF. La Autoexigencia (8 ítems), informa de la actitud perfeccionista con la que el niño se enfrenta a sus tareas (e.g., "No me gusta ser ni el segundo, quiero ser el primero"). La Presión externa (8 ítems) hace referencia a que el niño percibe su medio ambiente próximo como demandante de conductas perfectas (e.g., "Mis padres o profesores me castigan o riñen cuando no hago las cosas perfectamente"). Se incluyó la raíz "Piensa en cómo te comportas y en lo que sientes en relación a las clases de Educación Física y elige una respuesta" para contextualizarlo a las clases de esta materia. Se utilizó una escala Likert de 5 puntos, siendo 1= "muy en desacuerdo" a 5 = "muy de acuerdo".

Afectividad. Se utilizó la versión del PANAS (Positive and Negative Affect Schedule) validada en castellano por Sandín (2003) para su uso con niños y adolescentes (PANASN) en el contexto de la EF. Se compone de 20 ítems y presenta una estructura bidimensional: afecto positivo (e.g., "me intereso por la gente o las cosas") y afecto negativo (e.g., "siento miedo"), con 10 ítems para cada subescala. Se incluyó la raíz "Indica cómo te sueles sentir en clase de 
Méndez-Giménez, A.; Cecchini-Estrada, J.A.; Fernández-Rio, J. (2015). Perfeccionismo, afectividad y satisfacción con la vida en educación física. RICYDE. Revista internacional de ciencias del deporte, 41(11), 297304. http://dx.doi.org/10.5232/ricyde2015.04107

Educación Física...”. Se utilizó una escala Likert de 5 puntos, siendo 1 = "muy en desacuerdo" a 5 = "muy de acuerdo".

Satisfacción con la vida. Se utilizó la Escala de Satisfacción con la Vida validada al castellano por Cabañero y col. (2004). El cuestionario mide un único factor y se compone de 5 ítems (e.g., "Las condiciones de mi vida son excelentes"). Se utilizó una escala de 5 puntos siendo 1 "muy en desacuerdo" y 5 "muy de acuerdo".

\section{Procedimiento}

Se obtuvo el permiso de los centros educativos y el consentimiento paterno de los participantes. Los estudiantes completaron los tres cuestionarios a la vez y de manera anónima, empleando aproximadamente unos 20 minutos. Los investigadores aseguraron a los estudiantes que sus respuestas no afectarían a sus notas.

Análisis de datos

Se realizaron análisis exploratorios y descriptivos, correlaciones bivariadas y se calcularon los Alfas de Cronbach (SPSS.18). Para poner a prueba el modelo de la hipótesis se realizó un path análisis mediante el programa EQS, 6.2. El path análisis o análisis del camino es un método multivariante que permite identificar la contribución directa e indirecta de un conjunto de variables independientes para explicar la variabilidad de las variables dependientes. El análisis previo de los datos reveló una curtosis multivariante (3.40) que no permite aceptar la normalidad multivariante, por este motivo se realizó un análisis basado en el estadístico Satorra-Bentler chi-cuadrado $\left(\mathrm{S}-\mathrm{B} \chi^{2}\right)$ y en los estimadores estándar robustos, ya que sirven como corrección para $\chi^{2}$ cuando las suposiciones de distribución son violadas (Byrne, 2008).

La evaluación de la bondad del ajuste de los datos se determinó mediante criterios múltiples: como índices de ajuste incremental se usó el *CFI (Comparative Fit Index), como medida de los índices de ajuste absoluto se utilizó el *RMSEA (Root Mean Square Error Aproximation) y el SRMR (Root Mean Square Residual). El *CFI representa la versión robusta del CFI que se calcula en base al estadístico $\mathrm{S}-\mathrm{B} \chi^{2}$ y se sugieren un valor de .95 como indicativo de buen ajuste. En el *RMSEA los valores inferiores a .05 indican un buen ajuste, y valores de hasta .08 representan errores razonables de aproximación. El análisis se completó con el intervalo de confianza al $90 \%$ proporcionado por el *RMSEA, y la SRMR (valores inferiores a .08 son indicativos de un buen ajuste).

\section{Resultados}

\section{Análisis descriptivos}

La Tabla 1 presenta las medias, desviaciones típicas, los coeficientes alfa de Cronbach y correlaciones para las variables utilizadas en este estudio. Los valores más altos se dan en la satisfacción con la vida y el afecto positivo, mientras los más bajos recaen en la presión externa y el afecto negativo. La satisfacción con la vida se relaciona positivamente con autoexigencia y afecto positivo y, negativamente, con presión externa y afecto negativo. 
Méndez-Giménez, A.; Cecchini-Estrada, J.A.; Fernández-Rio, J. (2015). Perfeccionismo, afectividad y satisfacción con la vida en educación física. RICYDE. Revista internacional de ciencias del deporte, 41(11), 297304. http://dx.doi.org/10.5232/ricyde2015.04107

Tabla 1. Medias, desviaciones típicas, correlaciones bivariadas y alfas de Cronbach (diagonal)

\begin{tabular}{lccccccc}
\hline & $M$ & $D T$ & 1 & 2 & 3 & 4 & 5 \\
\hline 1. Auto-exigencia & 3.20 & .76 & .72 & & & & \\
2. Presión externa & 2.03 & .88 & $.44^{* *}$ & .87 & & & \\
3. Afecto positivo & 3.45 & .65 & $.36^{* *}$ & .07 & .80 & & \\
4. Afecto negativo & 2.03 & .81 & $.19^{* *}$ & $.34^{* *}$ & -.09 & .87 & \\
5. Satisfacción con la vida & 3.49 & .98 & $.15^{* *}$ & $-.31^{* *}$ & $.31^{* *}$ & $-.24^{* *}$ & .88 \\
\hline
\end{tabular}

${ }^{*} p<.05 \stackrel{* *}{p}<.01$

\section{Análisis de rutas}

Los resultados mostraron que los índices de ajuste presentan valores aceptables: S-B $\chi^{2}(2)=$ 7.32, $p=.112 ; * \mathrm{CFI}=.99 ; * \mathrm{RMSEA}(90 \% \mathrm{CI})=.05(.000-.093) ; \mathrm{SRMR}=.02$.

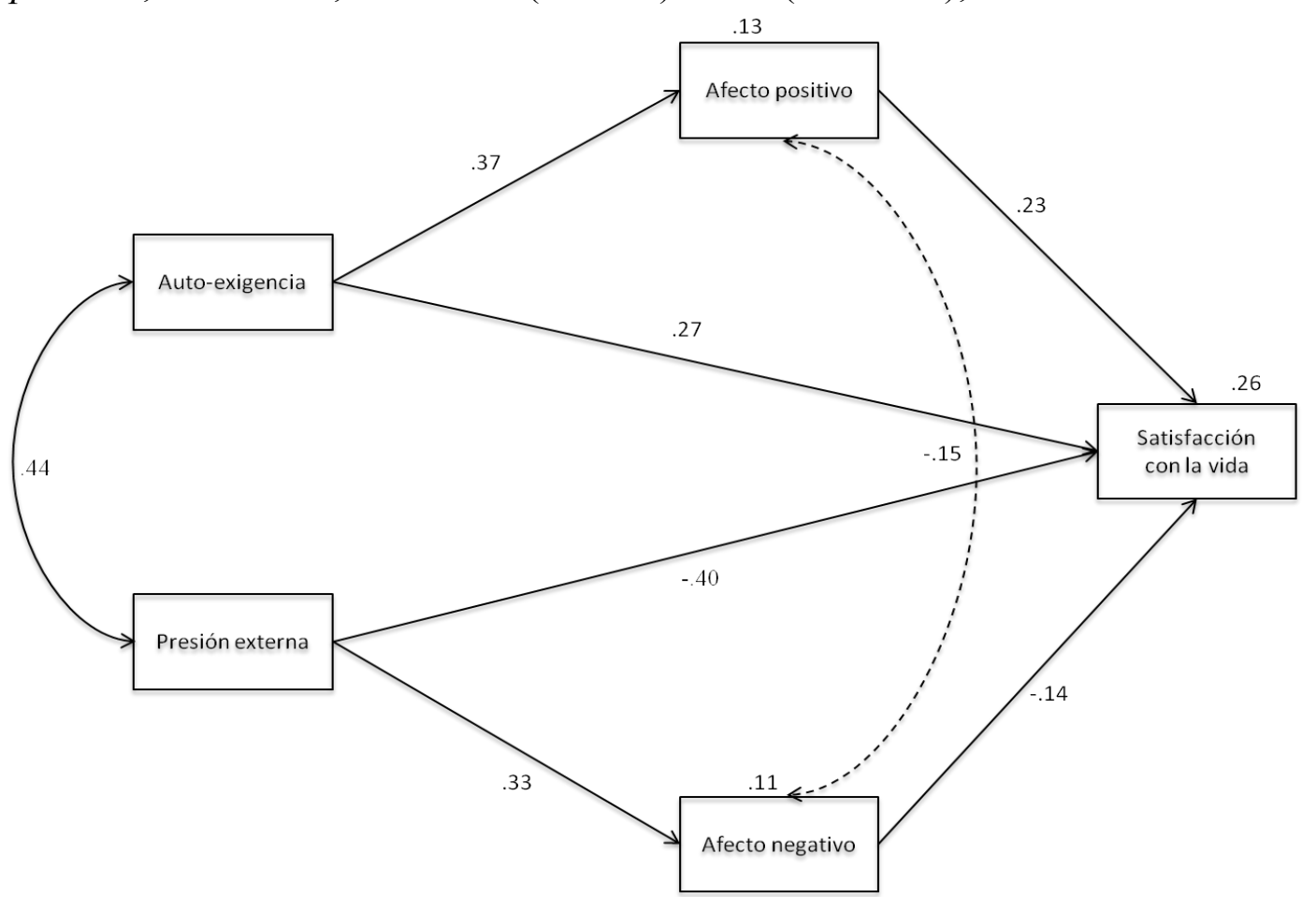

Figura 1. Modelo de rutas analizado de perfeccionismo, afectividad y satisfacción con la vida

\section{Discusión}

De manera consistente con la literatura, las medidas del perfeccionismo predijeron de manera contrapuesta las dimensiones de afecto y satisfacción con la vida en los estudiantes de EF. La autoexigencia predijo positivamente la satisfacción con la vida. Los adolescentes que mantenían altos estándares personales percibieron su experiencia vital de manera muy positiva. Además, mediante una segunda ruta, la autoexigencia predijo directa y positivamente el afecto positivo, que a su vez medió la relación con la satisfacción con la vida. Los adolescentes que establecen altos estándares se muestran más vitales, entusiastas, orgullosos, activos e interesados por emprender acciones o relacionarse con otras personas. Dicho estado afectivo positivo produce una mayor satisfacción con las condiciones de vida de que se dispone. Estos resultados son congruentes con varios estudios realizados, tanto con población adulta como adolescente, que han empleado medidas de perfeccionismo similares 
Méndez-Giménez, A.; Cecchini-Estrada, J.A.; Fernández-Rio, J. (2015). Perfeccionismo, afectividad y satisfacción con la vida en educación física. RICYDE. Revista internacional de ciencias del deporte, 41(11), 297304. http://dx.doi.org/10.5232/ricyde2015.04107

en contextos culturales diversos (Gilman y col., 2005; Öngen, 2009; Stoeber y Otto, 2006) y ayudan a entender cómo se produce esta relación.

Por otro lado, la presión externa ejercida por padres y profesores predijo negativamente (en una primera ruta) la satisfacción con la vida, y en una segunda ruta, positivamente el afecto negativo (Stoeber y Otto, 2006), y negativamente, la satisfacción con la vida. Aquellos adolescentes sensibles a un entorno que les demanda ser perfectos, preocupados por satisfacer los requerimientos de terceros sufren constantes tensiones, sensación de estrés, sentimientos de culpabilidad, enfado, mal humor, vergüenza y nerviosismo, lo que les lleva a una visión más pesimista de la vida. Con mayor probabilidad una fuerte presión externa percibida para alcanzar la excelencia (i.e., académica) pone en riesgo a los adolescentes de padecer síntomas emocionales severos. Los padres, docentes, orientadores y psicólogos deberían ser cautos a la hora de presionar a los adolescentes, valorar exactamente el tipo de perfeccionismo del que disponen, y ayudarles a gestionar ambas dimensiones del perfeccionismo. Cuando los adolescentes adoptan conductas perfeccionistas autoexigentes el consejo es que se mantengan en ellas, sin ser demasiado autocríticos si no alcanzan los estándares deseados. Si adoptan la dimensión presión externa se les debería ayudar a evaluarse de manera más realista para asumir estándares más acordes a su competencia (Öngen, 2009). Futuras investigaciones deberían examinar esas relaciones con sujetos de estatus social diverso y rango de edad más joven, lo que permitiría comprender cómo se produce la transición del perfeccionismo de la infancia a la adolescencia y sus relaciones con la salud.

\section{Conclusiones}

Estos resultados reafirman la existencia de una dimensión saludable de perfeccionismo, que promueve la calidad de vida, el bienestar subjetivo, y una dimensión insana (no internalizada o regulada externamente) que conlleva niveles más bajos de felicidad (Ofoghi y Besharat, 2010) en el contexto de la EF. Los resultados de esta investigación tienen implicaciones didácticas: el profesorado de EF debería generar climas que favorezcan conductas autoexigentes entre los estudiantes ajustadas a sus potencialidades, y a la vez evitar presiones excesivas que puedan frustrar las expectativas en caso de no alcanzar los estándares deseados. Entre las limitaciones del estudio señalamos la naturaleza transversal del diseño empleado, así como que los participantes pertenecían a un número limitado de centros educativos. Futuros trabajo deberían comprobar el modelo en otras muestras de estudiantes y contemplar diferentes franjas de edad.

\section{Referencias}

Arana, F.G. (2003). Fobia social como problema de alto perfeccionismo: Estudio de caso aplicando un tratamiento cognitivo conductual. Revista Argentina de Clínica Psicológica, 11(3), 191-209.

Bieling, P.J.; Israeli, A.L.; Smith, J., \& Antony, M.M. (2003). Making the grade: The behavioral consequences of perfectionism in the classroom. Personality and Individual Differences, 35, 163-178. http://dx.doi.org/10.1016/S0191-8869(02)00173-3

Byrne, B.M. (2008). Testing for multigroup equivalence of a measuring instrument: $A$ walk through the process. Psicothema, 20(4), 872-882. 
Méndez-Giménez, A.; Cecchini-Estrada, J.A.; Fernández-Rio, J. (2015). Perfeccionismo, afectividad y satisfacción con la vida en educación física. RICYDE. Revista internacional de ciencias del deporte, 41(11), 297304. http://dx.doi.org/10.5232/ricyde2015.04107

Cabañero, M.J.; Richart, M.; Cabrero, M.; Orts, J.; Reig, A., y Tosal, B (2004). Fiabilidad y validez de la Escala de Satisfacción con la Vida de Diener en una muestra de mujeres embarazadas y puérperas. Psicothema, 16(3), 448-455.

Chang, E.C. (2002). Examining the link between perfectionism and psychological maladjustment: social problem solving as a buffer. Cognitive Therapy and Research, 26(5), 581-595. http://dx.doi.org/10.1023/A:1020329625158

Cockell, S.J.; Hewitt, P.L.; Seal, B.; Sherry, S.; Goldner, E.M.; Flett, G.L., \& Remick, R. A. (2002). Trait and self-presentational dimensions of perfectionism among woman with anorexia nervosa. Cognitive Therapy and Research, 26(6), 745-758. http://dx.doi.org/10.1023/A:1021237416366

Einstein, D.A.; Lovibond, P.F., \& Gaston, J.E. (2000). Relationship between perfectionism and emotional symptoms in an adolescent sample. Australian Journal Psychology, $52,89-93$. http://dx.doi.org/10.1080/00049530008255373

Frost, R.O.; Heimberg, R.G.; Holt, C.S.; Mattia, J.I., \& Neubauer, A.L. (1993). A comparison of two measures of perfectionism. Personality and Individual Differences, 14, 119-126. http://dx.doi.org/10.1016/0191-8869(93)90181-2

Frost, R.O.; Marten, P.; Lahart, C., \& Rosemblate, R. (1990). The dimensions of perfectionism. Cognitive Therapy and Research, 14, 449-468. http://dx.doi.org/10.1007/BF01172967

Gaudreau, P., \& Thompson, A. (2010). Testing a $2 \times 2$ model of dispositional perfectionism. Personality and Individual Differences, 48, 532 - 537. http://dx.doi.org/10.1016/j.paid.2009.11.031

Gilman, R.; Ashby, J. S.; Sverko, D.; Florell, D., \& Varjas, K. (2005). The relationship between perfectionism and multidimensional life satisfaction among Croatian and American youth. Personality and Individual Differences, 39, 155. http://dx.doi.org/10.1016/j.paid.2004.12.014

Hewitt, P.L., \& Flett, G.L. (2002). Perfectionism. Washington, DC: American Psychological Association.

Hewitt, P.L., \& Flett, G.L. (1991). Perfectionism in the self and social contexts: Conceptualization, assessment, and association with psychopathology. Journal of Personality and Social Psychology, 60, 456-470. http://dx.doi.org/10.1037/0022-3514.60.3.456

Hewitt, P.L.; Caelian, C.F.; Flett, G.L.; Sherry, S.B.; Collins, L., \& Flynn C.A. (2002) Perfectionism in children: Associations with depression, anxiety, and anger. Personality and Individual Differences, 32, 1049-1061. http://dx.doi.org/10.1016/S0191-8869(01)00109-X

Kawamura, K.Y.; Hunt, S.L.; Frost, R.O., \& DiBartolo, P. (2001). Perfectionism, Anxiety, and Depression: Are the Relationships Independent? Cognitive Therapy and Research, 25(3), 291-301. http://dx.doi.org/10.1023/A:1010736529013

Lozano, L.M.; García, E.; Martín, M., y Lozano, L. (2012). Desarrollo y validación del Inventario de Perfeccionismo Infantil (IPI). Psicothema, 24(1), 149-155.

Méndez-Giménez, A.; Cecchini-Estrada, J.A., y Fernandez-Río, J. (2015). Perfeccionismo, metas de logro $2 \times 2$ y regulaciones motivacionales en el contexto de la educación física. Aula Abierta, 43(1), 18-25.

http://dx.doi.org/10.1016/j.aula.2014.09.001 
Méndez-Giménez, A.; Cecchini-Estrada, J.A.; Fernández-Rio, J. (2015). Perfeccionismo, afectividad y satisfacción con la vida en educación física. RICYDE. Revista internacional de ciencias del deporte, 41(11), 297304. http://dx.doi.org/10.5232/ricyde2015.04107

Nounopoulos, A.; Ashby, J.S., \& Gilman, R. (2006). Coping resources, perfectionism, and academic performance among adolescents. Psychology in the Schools, 43, 613-622. http://dx.doi.org/10.1002/pits.20167

Ofoghi, Z., \& Besharat, M.A. (2010). Perfectionism and physical ill-health. Procedia Social and Behavioral Sciences, 5, 1119-1123. http://dx.doi.org/10.1016/j.sbspro.2010.07.246

Öngen, D.E. (2009). The relationship between perfectionism and multidimensional life satisfaction among high school adolescents in Turkey. Journal of Multicultural Counseling and Development, 37, 52-64.

http://dx.doi.org/10.1002/j.2161-1912.2009.tb00091.x

Rice, K.G., \& Preusser, K.J. (2002). The Adaptive/Maladaptive Perfectionism Scale. Measurement and Evaluation in Counseling and Development, 34(4), 210-222.

Sandín, B. (2003). Escalas PANAS de afecto positivo y negativo para niños y adolescentes (PANASN). Revista de Psicopatología y Psicología Clínica, 8(2), 173182. http://dx.doi.org/10.5944/rppc.vol.8.num.2.2003.3953

Stoeber, J., \& Childs, J.H. (2011). Perfectionism. En: R.J.R. Levesque (Ed.), Encyclopedia of adolescence Vol. 4, Part 16 (pp. 2053-2059). New York: Springer.

Stoeber, J., \& Otto, K. (2006). Positive conceptions of perfectionism: Approaches, evidence, challenges. Personality and Social Psychology Review, 10, 295-319. http://dx.doi.org/10.1207/s15327957pspr1004_2

Stoeber, J., \& Rambow, A. (2007). Perfectionism in adolescent school students: Relations with motivation, achievement, and well-being. Personality and Individual Differences, 42(7), 1379-1389.

http://dx.doi.org/10.1016/j.paid.2006.10.015

Stoeber, J., \& Stoeber, F.S. (2009). Domains of perfectionism: Prevalence and relationships with perfectionism, gender, age, and satisfaction with life. Personality and Individual Differences, 46, 530-535.

http://dx.doi.org/10.1016/j.paid.2008.12.006 